\title{
AN EXAMPLE OF AN OPEN RIEMANN SURFACE NOT UNIFORMLY LARGE WITH RESPECT TO GREEN'S FUNCTIONS
}

\author{
By MASAHIKo TANIGUCHI
}

\section{§1. Introduction and the main result.}

Let $R$ be an open Riemann surface. To assure certain uniformness of $R$, we may impose the following conditions on $R$.

(G) Assuming that $R$ admits Green's functions $g(\cdot, q ; R)$ with the pole $q \in R$, there is a positive constant $M$ such that $\{p \in R: g(p, q ; R)>M\}$ is simply connected for every $q \in R$.

(H) Letting $d_{R}(\cdot, \cdot)$ be Poincaré's hyperbolic distance on $R$, there is a positive $\varepsilon$ such that $\left\{p \in R: d_{R}(p, q)<\varepsilon\right\}$ is simply connected for every $q \in R$.

A surface satisfying $(\mathrm{H})$ is called one with a positive injectivity radius and has several nice properties (cf. [4]). The condition $(G)$ is recently considered in [3] and [7].

Remark 1. The condition $(\mathrm{G})$ implies $(\mathrm{H})$. In fact, let $F_{p}$ be a Fuchsian group acting on $\{|z|<1\}$ and corresponding to a universal covering map $\pi_{p}$ of $\{|z|<1\}$ to a Riemann surface $R$ satisfying $(G)$ such that $\pi_{p}(0)=p$ for arbitrarily given $p \in R$. Then since $g\left(\pi_{p}(z), p ; R\right)=\Sigma_{f \in F_{p}} \log |1 / f(z)|, \pi_{p}$ is injective on the disk $\{z: \log |1 / z|>M\}$, which has some hyperbolic radius depending only on $M$.

Remark 2. In case of finite surfaces, $(G)$ is equivalent to $(H)$. In fact, in this case each of $(\mathrm{G})$ and $(\mathrm{H})$ is equivalent to the condition for non-existence of punctures.

But in general, $(H)$ does not necessarily implies $(G)$. Actually, the purpose of this note is to show the following.

THEOREM. There is a regular Riemann surface of Parreau-Widom type which satisfies $(H)$ but not $(G)$.

Here for regular Riemann surfaces of Parreau-Widom type, see for example [5]. We will construct a family of Riemann surfaces satisfying $(\mathrm{H})$ but not $(\mathrm{G})$ in $\S 2$, and give a proof of Theorem in $\S 3$.

Received November 19, 1986 
Finally, the author would like to thank the referee for his helpful advices.

\section{$\S 2$. Construction.}

Let $S_{1}$ be a compact bordered Riemann surface of genus one whose border consists of a single geodesic $d_{1}$. Namely, we have a Riemann surface $R_{1}$ of genus one with one hole and a bordered subsurface $S_{1}$ (called the Nielsen kernel, cf. [2]) of $R_{1}$ such that $R_{1}-S_{1}$ is a doubly connected region and the border of $S_{1}$ is a compact simple geodesic in $R_{1}$ (with respect to Poincaré's hyperbolic metric on $R_{1}$ ). Let $c$ be the length of $d_{1}$ and $S_{0}$ be a compact bordered Riemann surface of genus one whose border consists of two closed geodesics $d_{0}$ and $d_{0}^{\prime}$ with the same length $c$. Also we set $W_{1}=R_{1}-\left(S_{1}-d_{1}\right)$.

For every $n \geqq 2$, we can construct inductively a compact bordered Riemann surface $S_{n}$ from $S_{n-1}$ and $S_{0}$ by gluing the border $d_{n-1}$ of $S_{n-1}$ to $d_{0}$ isometrically so that the hyperbolic metrics on $S_{n-1}$ and $S_{0}$ coincide with that on $S_{n}$. And for every $n$, let $R_{n}$ be a Riemann surface (called the Nielsen extension of $S_{n}$, cf. [2]) obtained from $S_{n}$ and $W_{1}$ by gluing $d_{n}$ and $d_{1}$ considered as the border of $W_{1}$. In the sequel, we denote by $S_{1, n}$ and $W_{1, n}$ the parts of $R_{n}$ corresponsing to $S_{1}$ and $W_{1}$ in $R_{n}$, respectively.

Also fix a point $p_{1}$ in $S_{1}$ and, denote by $p_{n}$ the point on $S_{1, n}$ corresponding to $p_{1}$ for every $n$.

Here recall that $W_{1}$ can be represented as the quotient bordered surface of $\Delta=\{z: \operatorname{Im} z>0, \operatorname{Re} z \geqq 0\}$ by the elementary group generated by $A(z)=a z$, where $a>1$ and then is determined by $c$. For every $n$ and positive $e<\left(a^{1 / 2}-1\right) / 2$, we denote by $B_{n}(e)$ and $B_{n}^{\prime}(e)$ the subregions of $W_{1, n}$ corresponding to $\{z \in \Delta$ : $|z-1|<e\}$ and $\left\{z \in \Delta:\left|z-a^{1 / 2}\right|<e \cdot a^{1 / 2}\right\}$, respectively. Note that $B_{n}(e)$ and $B_{n}^{\prime}(e)$ are mutually disjoint. Let $b_{n}(e)$ and $b_{n}^{\prime}(e)$ be the relative boundaries of $B_{n}(e)$ and $B_{n}^{\prime}(e)$ in $W_{1, n}$, respectively.

Then for any given sequence $\left\{e_{n}\right\}_{n=1}^{\infty}$ of positive numbers $e_{n}$ with $e_{n}<$ $\left(a^{1 / 2}-1\right) / 2$, we can construct a sequence $\left\{T_{n}\left(e_{n}\right)\right\}_{n=0}^{\infty}$ of Riemann surfaces, where we set $T_{0}\left(e_{0}\right)=R_{1}$, inductively as follows.

Set $T_{0}\left(e_{1}\right)^{*}=R_{1}-B_{1}^{\prime}\left(e_{1}\right)$ and $R_{2}\left(e_{1}\right)^{*}=R_{2}-B_{2}\left(e_{1}\right)$, and glue $b_{1}^{\prime}\left(e_{1}\right)$ to $b_{2}\left(e_{1}\right)$ by the mapping corresponding to $f(z)=-e_{1}^{2} \cdot a^{1 / 2} /\left(z-a^{1 / 2}\right)+1$. Then as before we have a Riemann surface $T_{1}\left(e_{1}\right)$ having the curve $c_{1}\left(e_{1}\right)$ resulting from $b_{1}^{\prime}\left(e_{1}\right)$ and $b_{2}\left(e_{1}\right)$ as a geodesic (with respect to the hyperbolic metric on $T_{1}\left(e_{1}\right)$ ). Next suppose that we have constructed $\left\{T_{n}\left(e_{n}\right)\right\}_{n=1}^{k-1}$. Then set $T_{k-1}\left(e_{k}\right)^{*}=T_{k-1}\left(e_{k-1}\right)$ $-B_{k}^{\prime}\left(e_{k}\right)$ and $R_{k+1}\left(e_{k}\right)^{*}=R_{k+1}-B_{k+1}\left(e_{k}\right)$, and glue $b_{k}^{\prime}\left(e_{k}\right)$ to $b_{k+1}\left(e_{k}\right)$ similarly as above. We denote by $T_{k}\left(e_{k}\right)$ the resulting Riemann surface. See Figure below.

Now since $T_{n}\left(e_{n+1}\right) *$ can be considered as a bordered subsurface of $T_{n+1}\left(e_{n+2}\right) *$ for every $n$, we can consider a Riemann surface $R=\bigcup_{n=1}^{\infty} T_{n}\left(e_{n+1}\right) *$ as the inductive limit of $\left\{T_{n}\left(e_{n+1}\right)^{*}\right\}_{n=1}^{\infty}$. And we have the following.

Proposition 1. For every sequence $\left\{e_{n}\right\}_{n=1}^{\infty}$ of positive numbers $e_{n}$ such that $e_{n}<\left(a^{1 / 2}-1\right) / 2$, the surface $R=\bigcup_{n=1}^{\infty} T_{n}\left(e_{n+1}\right)^{*}$ satisfies $(H)$ but not $(G)$. 


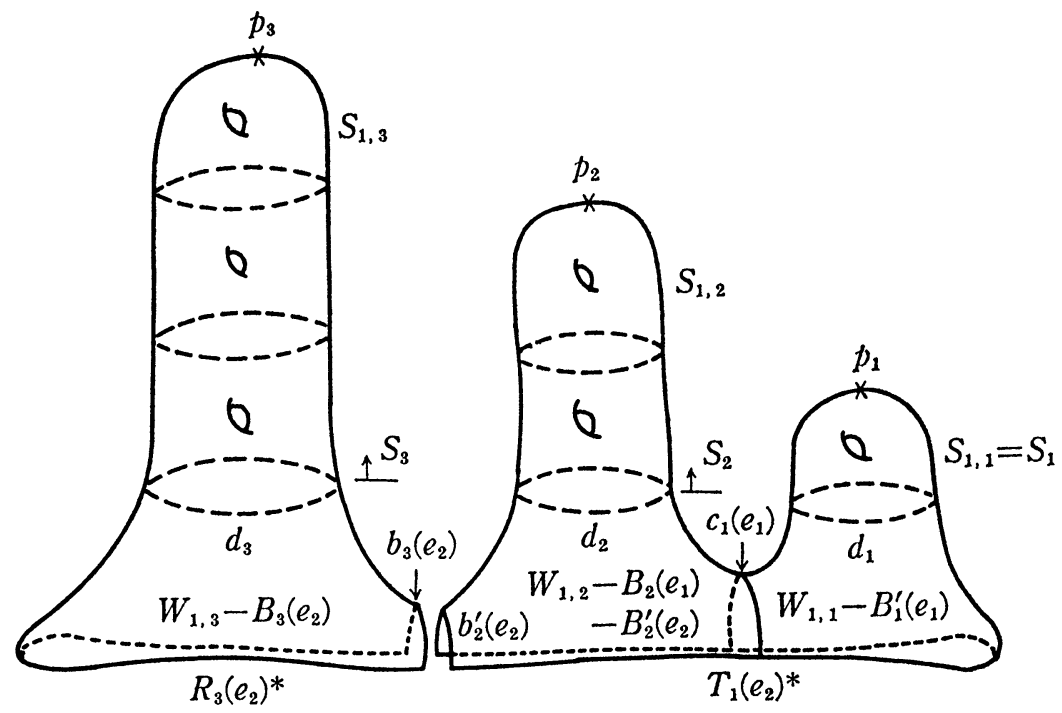

[Figure] The parts of the surface $T_{2}\left(e_{2}\right)$.

Proof. It is clear from the above construction that $R$ satisfies $(\mathrm{H})$ and that $R$ admits Green's functions.

Next let $S=\bigcup_{n=1}^{\infty} S_{n}$ be the inductive limit of $\left\{S_{n}\right\}_{n=1}^{\infty}$. Then $\left\{S_{n}-d_{n}\right\}_{n=1}^{\infty}$ gives a canonical exhaustion of $S$. Also from the construction we can see by Nevanlinna's modular test (cf. [1, IV. 15D]) that $S$ admits no Green's functions, or equivalently, that $g\left(\cdot, p_{1} ; S_{n}-d_{n}\right)$ tends to $+\infty$ locally uniformly on $S$ as $n$ tends to $+\infty$, where we regard $p_{1}$ as a point on $S$.

Since $g\left(\cdot, p_{n} ; R\right)>g\left(\cdot, p_{1} ; S_{n}-d_{n}\right)$ on $S_{n}-d_{n}$ considered as a subsurface of $R$ (where $p_{1}$ is identified with $p_{n}$ ) for every $n$, we can find, for any given $M$, an $N$ such that $\left\{p \in R: g\left(p, p_{N} ; R\right)>M\right\}$ contains $S_{1, N}$, hence is not simply connected.

Thus we conclude that $R$ does not satisfy $(G)$.

q.e.d.

\section{$\S 3$. Proof of Theorem.}

First we will show the following

PROPOSITION 2. Let $\left\{t_{n}\right\}_{n=1}^{\infty}$ and $\left\{e_{n}\right\}_{n=1}^{\infty}$ be two sequences of positive numbers which satisfy the following conditions; for every $n$, it holds that

1) $t_{n}<\min \left\{t_{n-1}, 1 / n^{3}\right\}$ and $e_{n}<\left(a^{1 / 2}-1\right) / 2$,

2) $D_{n}=\left\{p \in T_{n-1}\left(e_{n-1}\right): g_{1, n-1}>4 t_{n}\right\}$ is homeomorphic to $T_{n-1}\left(e_{n-1}\right)$ and contains $D_{n-1}$,

3) $g_{1, n-1}<t_{n} / 2$ on $\partial T_{n-1}\left(e_{n}\right)^{*}=b_{n}^{\prime}\left(e_{n}\right)$, and

4) $\left|g_{1, n}-g_{1, n-1}\right|<t_{n} / 2^{n+1}$ on $T_{n-1}\left(e_{n}\right)^{*}$, 
where we set $t_{0}=1, D_{0}=\varnothing$ and $g_{1, n}=g\left(\cdot, p_{1} ; T_{n}\left(e_{n}\right)\right)$ for every $n$.

Then $R=\bigcup_{n=1}^{\infty} T_{n}\left(e_{n+1}\right) *$ is a regular Riemann surface of Parreau-Widom type.

Proof. To show that $R$ is regular, first not that, by 4$), g_{1, n}$ converges locally uniformly on $R-\left\{p_{1}\right\}$ to a positive harmonic function, say $h$, as $n$ tends to $+\infty$. Moreover, 1), 3) and 4) imply that

5) $\left|h-g_{1, n-1}\right| \leqq \sum_{m=n}^{\infty}\left|g_{1, m}-g_{1, m-1}\right|<\sum_{m=n}^{\infty} t_{m} / 2^{m+1}<t_{n} / 2^{n}$ on $T_{n-1}\left(e_{n}\right)^{*}$, and

6) $h \leqq \sum_{m=n}^{\infty}\left|g_{1, m}-g_{1, m-1}\right|+g_{1, n-1}<t_{n} / 2^{n}+t_{n} / 2 \leqq t_{n}$ on $\partial T_{n-1}\left(e_{n}\right)^{*}$, for every $n$.

Hence by the maximal principle, for every $\varepsilon>0$, we can find a compact set $F$ in $R$ such that $h<\varepsilon$ on $R-F$, which implies that $h=g\left(\cdot, p_{1} ; R\right)$ and that $R$ is a regular Riemann surface.

Next recall that a regular Riemann surface $R$ is of Parreau-Widom type if and only if

$$
\sum_{q \in Z} g\left(q, p_{1} ; R\right)<+\infty,
$$

where $Z$ is the set of all critical points of $g\left(\cdot, p_{1} ; R\right)$ including multiplicity (cf. [5, V. 1C Theorem]).

Fix $n$ arbitrarily. Since $L_{n}=\left\{p \in R: g\left(p, p_{1} ; R\right)=2 t_{n}\right\}$ is contained in $T_{n-1}\left(e_{n}\right)^{*}-\bar{D}_{n}$ by 4$\left.), 5\right)$ and 6$)$, and since $T_{n-1}\left(e_{n}\right)^{*}-\bar{D}_{n}$ is a doubly connected region by 2$)$, we see that $L_{n}$ is a simple closed analytic curve and $D_{n}^{\prime}=\{p \in R$ : $\left.g\left(p, p_{1} ; R\right)>2 t_{n}\right\}$ is homeomorphic to $T_{n-1}\left(e_{n-1}\right)$.

Here it is well known (as a corollary of Riemann-Roch theorem, cf. [1, V. 27A]) that $D_{n}^{\prime}$ contains exactly $2 \cdot \sum_{k=1}^{n} k=n(n+1)$ critical points of $g\left(\cdot, p_{1} ; R\right)$. Hence $D_{n}^{\prime}-D_{n-1}^{\prime}$ contains $2 n$ such points and it holds that

$$
\sum_{q \in Z} g\left(q, p_{1} ; R\right) \leqq \sum_{q \in Z \cap D_{1}^{\prime}} g\left(q, p_{1} ; R\right)+\sum_{n=1}^{\infty} 4(n+1) t_{n},
$$

which is finite by 1 ). Thus we conclude that $R$ is of Parreau-Widom type.

q.e.d.

Thus to complete the proof of Theorem, it remins to give such sequences $\left\{t_{n}\right\}_{n=1}^{\infty}$ and $\left\{e_{n}\right\}_{n=1}^{\infty}$ as in Proposition 2.

For this purpose, fix $n$ arbitrarily, and suppose that $\left\{t_{k}\right\}_{k=1}^{n-1}$ and $\left\{e_{k}\right\}_{k=1}^{n-1}$ are determined. Then take $t_{n}$ so small that 1) and 2) in Proposition 2 hold, and then take $e_{n, 0}$ so small that $g_{1, n-1}<t_{n} / 2^{n+2}$ on $b_{n}^{\prime}\left(e_{n, 0}\right)$. Next set $E=\{p \in$ $\left.T_{n-1}\left(e_{n-1}\right): g_{1, n-1}(p) \geqq t_{n} / 2^{n+2}\right\}$, then $E$ is compact in $T_{n-1}\left(e_{n-1}\right)-\overline{B_{n}^{\prime}\left(e_{n, 0}\right)}$. And if we find an $e_{n}^{\prime}<e_{n, 0}$ such that

$$
\left|g\left(\cdot, p_{1} ; T_{n}\left(e_{n}^{\prime}\right)\right)-g_{1, n-1}\right|<t_{n} / 2^{n+2} \quad \text { on } E,
$$

we can conclude by the maximal principle that 3) and 4) hold with $e_{n}=e_{n}^{\prime}$.

Here the existence of such an $e_{n}^{\prime}$ follows by the fact that $g\left(\cdot, p_{1} ; T_{n}\left(e_{n}^{\prime}\right)\right)$ converges to $g_{1, n-1}$ locally uniformly on $T_{n-1}\left(e_{n-1}\right)$ as $e_{n}^{\prime}$ tends to 0 . This fact seems to be essentially well-known. But the author can not find any adequate reference, so we include a proof. 
For every $e_{n}^{\prime}<e_{n, 0}$, let $\hat{T}_{n}\left(e_{n}^{\prime}\right)$ be the double of $T_{n}\left(e_{n}^{\prime}\right)$ with respect to two ideal boundary arcs of $T_{n}\left(e_{n}^{\prime}\right)$ between $b_{n}^{\prime}\left(e_{n, 0}\right)$ and $b_{n+1}\left(e_{n, 0}\right)$. Then clearly, $\widehat{T}_{n}\left(e_{n}^{\prime}\right)$ admits Green's functions, and it holds that

$$
g\left(p, p_{1} ; T_{n}\left(e_{n}^{\prime}\right)\right)=g\left(p, p_{1} ; \hat{T}_{n}\left(e_{n}^{\prime}\right)\right)-g\left(p, p_{1}^{*} ; \hat{T}_{n}\left(e_{n}^{\prime}\right)\right)
$$

on $T_{n}\left(e_{n}^{\prime}\right)$ for every $e_{n}^{\prime}$, where $p_{1}^{*}$ is the mirror image of $p_{1}$. Since $\hat{T}_{n}\left(e_{n}^{\prime}\right)$ converges to a Riemann surface with one node corresponding to $e_{n}^{\prime}=0$ in the sence of the conformal topology as $e_{n}^{\prime}$ tends to 0 , we can see the assertion by [6, Corollary 1].

Now we have obtained $t_{n}$ and $e_{n}$ satisfying 1)-4) in Proposition 2. And by induction, we can show the existence of desired sequences, and finish the proof of Theorem.

q.e.d.

\section{REFERENCES}

[1] L. Ahlfors and L. Sario, Riemann surfaces, Princeton univ. Press 1960.

[2] L. BERs, Nielsen extensions of Riemann surfaces, Ann. Acad. Sci. Fenn. Ser. A. I. Math. 2 (1976), 29-34.

[3] Y. Goto, On BMO property for potentials on Riemann surfaces, J. Math. Kyoto Univ. 27 (1987), to appear.

[4] Y. Gото, Some extension property for BMO functions on Riemann surfaces, J. Math. Kyoto Univ., to appear.

[5] M. HAsum, Hardy classes on infinitely connected Riemann surfaces, Lecture Notes in Math. 1027, Springer, 1983.

[6] M. TANigUCHI, Continuity of certain differentials on finitely augmented Teichmüller spaces and variational formulas of Schiffer-Spencer's type, Tohoku Math. J. 38 (1986), 281-295.

[7] M. TANiguchi, A note on continuity of Green's functions on Riemann surfaces, Kodai Math. J. 10 (1987) to appear.

Department of Mathematics KYOTO UNIVERSITY 